\title{
Serum mesothelin and megakaryocyte potentiating factor in pancreatic and biliary cancers
}

\author{
Elad Sharon ${ }^{1, a}$, Jingli Zhang, ${ }^{1, a}$, Kevin Hollevoet ${ }^{1}$, \\ Seth M. Steinberg ${ }^{2}$, Ira Pastan ${ }^{1}$, Masanori Onda ${ }^{1}$, \\ Jochen Gaedcke ${ }^{3}$, B. Michael Ghadimi ${ }^{3}$, \\ Thomas Ried ${ }^{4}$ and Raffit Hassan ${ }^{1, *}$ \\ ${ }^{1}$ Laboratory of Molecular Biology, Center for Cancer \\ Research, National Cancer Institute, National Institutes of \\ Health, Bethesda, MD, USA \\ ${ }^{2}$ Biostatistics and Data Management Section, Center for \\ Cancer Research, National Cancer Institute, National \\ Institutes of Health, Bethesda, MD, USA \\ ${ }^{3}$ Department of General and Visceral Surgery, University \\ Medical Center Goettingen, Goettingen, Germany \\ ${ }^{4}$ Genetics Branch, Center for Cancer Research, National \\ Cancer Institute, National Institutes of Health, Bethesda, \\ MD, USA
}

\begin{abstract}
Background: Tumor mesothelin overexpression is present in different malignancies, including the majority of patients with pancreatic or biliary cancers. The objective of this study was to evaluate the use of shed serum mesothelin and megakaryocyte potentiating factor (MPF) concentrations as biomarkers for these cancers.

Methods: A total of 151 individuals, divided into five groups, were retrospectively analyzed: healthy donors $(n=15)$, patients with benign non-pancreatic conditions $(n=52)$, benign pancreatic conditions $(n=33)$, biliary carcinoma $(n=9)$, and pancreatic ductal adenocarcinoma $(n=42)$. Mesothelin and MPF concentrations were measured in serum with the Mesomark $^{\mathrm{TM}}$ and Human MPF ELISA, respectively.

Results: Mesothelin and MPF concentrations did not significantly differ among the five individual participant groups $(\mathrm{p}=0.34, \mathrm{p}=0.33$, respectively), nor did any other combination and pair-wise comparison of the participant groups demonstrated a significant difference in biomarker concentrations. In patients with pancreatic cancer, mesothelin or MPF concentrations were not associated with tumor stage $(\mathrm{p}=0.87, \mathrm{p}=0.48$, respectively) or differentiation grade ( $\mathrm{p}=0.73, \mathrm{p}=0.52$, respectively).
\end{abstract}

\footnotetext{
a Elad Sharon and Jingli Zhang are considered first authors for this publication and contributed equally.

*Corresponding author: Raffit Hassan, Laboratory of Molecular Biology, NCI, 37 Convent Drive, Room 5116, Bethesda, MD 20892-4264, USA

Phone: +1 301 451-8742, Fax: +1 301 402-1344,

E-mail: hassanr@mail.nih.gov

Received October 7, 2011; accepted November 14, 2011;

previously published online December 8, 2011
}

Conclusions: Serum mesothelin and MPF concentrations, measured with standard available ELISAs, were not specific for benign or pancreatic disease. Both biomarkers were not elevated in patients with pancreatic or biliary cancers, and consequently do not appear to be useful biomarkers for these malignancies.

Keywords: biliary cancer; megakaryocyte potentiating factor; mesothelin; pancreatic cancer.

\section{Introduction}

Pancreatic cancer is the fourth-leading cause of cancer mortality in the US, with an incidence of 43,140 cases and 36,800 deaths estimated in 2010 (1). Biliary cancer is markedly less common, with approximately 9000 new cases in the US per year. Because of the non-specific symptoms of pancreaticobiliary cancer, the vast majority of patients have advanced disease at presentation $(2,3)$. In patients with pancreatic cancer, fewer than $20 \%$ present with localized disease amenable to surgical resection. In these patients, median survival is around 20 months, while in those with unresectable disease, prognosis is dismal (3). Patients with biliary carcinoma frequently experience a relapse, despite surgery (2). In those with advanced disease, a combination treatment of cisplatin and gemcitabine results in a median survival of approximately 12 months (4).

As early diagnosis of a still localized tumor is thought to improve patient outcome, there is a critical need for tools that shorten the diagnostic delay. Due of their relative noninvasiveness and low cost, serum biomarkers are an attractive tool for such purpose. CA 19-9 is currently the only serum biomarker which demonstrated some clinical utility in pancreaticobiliary cancer, as a tool to help monitor disease activity (5). However, since CA 19-9 is usually not elevated in early stage patients, it is not useful in diagnosing early disease $(6,7)$.

Serum mesothelin and megakaryocyte potentiating factor (MPF) are promising blood protein biomarkers for mesothelioma and ovarian cancer (8-11). Both biomarkers originate from the mesothelin gene, which encodes a precursor protein that is cleaved into a soluble $31-\mathrm{kDa}$ fraction, MPF, and a membrane-bound 40-kDa glycoprotein, mesothelin (12). The latter can be cleaved and shed into the bloodstream together with MPF (13).

Membrane-bound mesothelin is normally present on the mesothelial cells lining the pleura, peritoneum and pericardium, but is highly expressed in several human cancers including mesothelioma, ovarian, and pancreaticobiliary 
cancers $(12,14,15)$. Given the presence of elevated mesothelin and MPF concentrations in mesothelioma and ovarian cancer (8-11), these biomarkers might also prove useful in pancreatic and biliary cancers. One recent study indeed described elevated serum mesothelin concentrations in benign and malignant pancreatic disease (16). That report, however, lacked a sufficient number of relevant controls, and the clinical value of both serum mesothelin and MPF in pancreatic cancers remains unclear.

The aim of this research study was to evaluate the value of serum mesothelin and MPF in pancreaticobiliary cancer, by comparing biomarker concentrations of these patients with those of other relevant control groups.

\section{Materials and methods}

\section{Study participants}

Between 2004 and 2006, patients were recruited at the Department of General and Visceral Surgery, University Medical Center Goettingen, Germany. Upon enrollment, patients were stratified into four groups according to diagnosis: 1) benign non-pancreatic conditions, 2) benign pancreatic conditions, 3) biliary carcinoma, and 4) pancreatic ductal adenocarcinoma. Blood sampling was done at diagnosis or prior to surgery, whenever relevant. Tumor staging of the patients with pancreatic cancer was done according to the American Joint Committee on Cancer staging guidelines (17). Specimens were collected under research protocols approved by an Institutional Review Board, and signed informed consent was obtained from all participants. In addition, serum samples from healthy donors were commercially obtained from Bioreclamation (East Meadow, NY, USA). All samples were kept at $-80^{\circ} \mathrm{C}$ until use.

\section{Biomarker assays}

Serum mesothelin and MPF concentrations were measured with the Mesomark ${ }^{\mathrm{TM}}$ ELISA (Fujirebio Diagnostics, Inc., Malvern, PA, USA) and the Human MPF ELISA (Medical \& Biological Laboratories Co., Ltd., Nagoya, Japan), respectively, following manufacturers' instructions $(18,19)$. Assays were run blinded to the sample data.

\section{Statistical analysis}

Continuous values were reported as median with 25 th and 75 th percentiles. Differences in mesothelin and MPF concentrations among groups of participants were evaluated with the Kruskal-Wallis and Wilcoxon rank sum tests. Groups were combined for analyses when results were found to be sufficiently similar statistically to permit this. Correlation between two parameters was evaluated with Spearman rank correlation analysis. All p-values are two-sided and reported without adjustment for multiple comparisons; a p-value of $<0.05$ was considered significant. Statistical analysis was done with SPSS version 17 (SPSS Inc., Chicago, IL, USA) and SAS version 8.2 (SAS Institute, Cary, NC, USA).

\section{Results}

\section{Participant characteristics}

A total of 151 participants was retrospectively collected (Table 1): 15 healthy donors; 52 patients with benign nonpancreatic condition, including hernia, diverticulitis, gallstones, Graves Disease and ileus; 33 patients with a benign pancreatic condition, including chronic pancreatitis, adenoma and acute pancreatitis; nine patients with biliary cancer; and 42 with pancreatic ductal adenocarcinoma. The majority of the patients with pancreatic cancer had stage III-IV disease at inclusion (Table 2). The healthy donors were significantly younger than each of the other patient groups $(\mathrm{p}<0.001)$ (Table 1).

\section{Biomarker concentrations are not elevated in pancreaticobiliary cancer}

The mesothelin and MPF concentrations of each of the five participant groups are presented in Table 1 and Figure 1. When using data from all 151 participants, mesothelin concentrations correlated moderately well with MPF concentrations $(\mathrm{r}=0.62 ; \mathrm{p}<0.001)$. Mesothelin and MPF concentrations did not significantly differ among the five individual participant groups $(\mathrm{p}=0.34, \mathrm{p}=0.33$, respectively). Furthermore, when comparing mesothelin and MPF concentrations of all 100 controls jointly with those of the 51 patients with pancreaticobiliary cancer, no significant difference was observed ( $\mathrm{p}=0.31, \mathrm{p}=0.16$, respectively). Not a single other combination and pair-wise comparison of participant groups demonstrated significant differences in biomarker concentrations (data not shown). However, when differentiating the 51 patients with pancreaticobiliary cancer from the 15 healthy donors, there was a trend towards higher mesothelin concentrations in the former group ( $\mathrm{p}=0.05)$. The difference in MPF concentrations between both groups displayed a similar trend, although less pronounced $(\mathrm{p}=0.11)$.

Table 1 Participant characteristics.

\begin{tabular}{lllll}
\hline Group & Number (female) & $\begin{array}{l}\text { Median age } \\
(\mathrm{P} 25-\mathrm{P} 75), \text { years }\end{array}$ & $\begin{array}{l}\text { Median mesothelin } \\
(\mathrm{P} 25-\mathrm{P} 75), \mathrm{nmol} / \mathrm{L}\end{array}$ & $\begin{array}{l}\text { Median MPF } \\
(\mathrm{P} 25-\mathrm{P} 75), \mathrm{ng} / \mathrm{mL}\end{array}$ \\
\hline Healthy donors & $15(11)$ & $31(23-39)$ & $0.58(0.15-0.72)$ & $8.33(5.20-10.81)$ \\
Benign non-pancreatic condition & $52(27)$ & $47(38-60)$ & $0.71(0.44-0.98)$ & $9.39(5.66-13.12)$ \\
Benign pancreatic condition & $33(13)$ & $59(51-68)$ & $0.69(0.47-0.95)$ & $7.52(5.30-11.67)$ \\
Biliary cancer & $9(6)$ & $64(63-76)$ & $0.71(0.53-1.18)$ & $7.93(6.69-14.65)$ \\
Pancreatic cancer & $42(21)$ & $67(60-71)$ & $0.66(0.52-0.94)$ & $8.94(6.57-13.43)$ \\
\hline
\end{tabular}

P25-75: 25th and 75th percentile. MPF, megakaryocyte potentiating factor. 
Table 2 Pancreatic cancer patient characteristics.

\begin{tabular}{lllll}
\hline Covariate & Stratification & Number & $\begin{array}{l}\text { Median mesothelin } \\
(\mathrm{P} 25-\mathrm{P} 75), \mathrm{nmol} / \mathrm{L}\end{array}$ & $\begin{array}{l}\text { Median MPF } \\
(\mathrm{P} 25-\mathrm{P} 75), \mathrm{ng} / \mathrm{mL}\end{array}$ \\
\hline Stage & IB-IIB & 17 & $0.76(0.49-1.01)$ & $8.74(6.39-12.45)$ \\
& III-IV & 25 & $0.65(0.54-0.89)$ & $9.14(6.74-16.34)$ \\
Tumor grade & $1-2$ & 21 & $0.67(0.51-0.98)$ & $8.74(6.06-13.10)$ \\
& 3 & 14 & $0.65(0.52-0.78)$ & $8.86(7.36-18.90)$ \\
& NOS & 7 & $0.83(0.45-1.17)$ & $9.14(5.71-20.91)$ \\
\hline
\end{tabular}

P25-75: 25th and 75th percentile. MPF, megakaryocyte potentiating factor, NOS, not otherwise specified.

\section{Biomarker concentrations do not differ according to pancreatic tumor stage or differentiation grade}

When stratifying patients with pancreatic cancer according to tumor stage (IB-IIB vs. III-IV), mesothelin and MPF concentrations did not differ significantly between both groups ( $\mathrm{p}=0.87, \mathrm{p}=0.48$, respectively) (Table 2). Similarly, when dividing these patients according to tumor grade (1-2 vs. 3), mesothelin and MPF concentrations did not differ significantly between the two groups $(\mathrm{p}=0.73, \mathrm{p}=0.52$, respectively) (Table 2).

\section{Discussion}

This study evaluated the use of serum mesothelin and MPF concentrations as biomarkers for pancreaticobiliary cancer. Biomarker concentrations were compared in patients with pancreatic and biliary cancers, benign pancreatic disease, benign non-pancreatic disease and healthy donors. Due to the retrospective nature of our study, these analyses were constrained by the number of available samples, but the study population was sufficiently large to obtain reliable results.

In our series, neither mesothelin nor MPF differentiated patients with pancreaticobiliary cancer from those with a benign pancreatic condition. For mesothelin, this was in agreement with a recent report by Johnston et al. (16), who compared concentrations in 74 patients with pancreatic cancer, five patients with benign pancreatic disease and five healthy donors. Interestingly and similar to the trend in our series, the five healthy individuals had significantly lower mesothelin values than the 74 patients with pancreatic cancer (16). The latter observation, although based on very small numbers, prompted Johnston et al. to state that mesothelin might be a useful biomarker for both benign and malignant pancreatic disease (16).

Our additional findings, however, do not support this statement. First, we found that mesothelin and MPF concentrations were unable to differentiate patients with a benign non-pancreatic disease from those with a benign or malignant pancreatic condition. This indicated that both biomarkers are not specific for pancreatic disease. Second, the observed trend towards higher biomarker concentrations in patients with pancreaticobiliary cancer, as compared to healthy donors, should be interpreted with caution. Mesothelin and MPF concentrations are indeed positively correlated with age
(20), and there was a large difference in age between those two groups. As such, the observed difference in biomarker concentrations was likely age-related, rather than associated with the presence of a tumor. It is possible that this was also the case in the study of Johnston et al. (16). This, however, cannot be verified, as the age of their five healthy individuals was not reported. Either way, from a clinical point of view, a diagnostic biomarker should primarily be able to differentiate individuals at risk, e.g., those with a benign disease, from those with cancer - which was not the case according to our data and that of Johnston et al. (16). Altogether, there is little evidence that mesothelin and MPF are useful for the diagnosis or screening of both benign and malignant pancreatic conditions.

Our data showed that the absence of any differential predictive capability for mesothelin and MPF was simply because patients with pancreaticobiliary cancer lacked elevated biomarker concentrations. The median mesothelin and MPF concentrations of our different participant groups were indeed similar to those of healthy controls in a previous report (10). The mesothelin concentrations we measured cannot be compared with those reported by Johnston et al., as they used a non-commercial version of the Mesomark ${ }^{\mathrm{TM}}$ ELISA (16).

Despite the absence of any diagnostic value, serum mesothelin and MPF could prove useful in other fields of pancreaticobiliary cancer management. A recent study, for example, indicated that the co-expression of mesothelin and CA125 is an indicator of poor outcome in pancreatic ductal carcinoma (21). In addition to prognosis, serum mesothelin and MPF concentrations have also shown to correlate with tumor stage and response to therapy in mesothelioma $(10,22,23)$. In our series, however, biomarker concentrations were not associated with pancreatic tumor burden or differentiation. This observation likely limits the use of serum mesothelin or MPF in any of the above-mentioned fields.

Further research should focus on the apparent discrepancy between the previously reported high mesothelin expression in pancreaticobiliary tumors and the absence of elevated mesothelin and MPF concentrations in serum. The latter is not consistent with findings in other mesothelinoverexpressing malignancies, including mesothelioma or ovarian cancer $(8-11,24)$. Possible explanations include the more limited tumor bulk in pancreaticobiliary cancers, and differences in the shedding mechanisms of mesothelin and MPF between malignancies. If the latter is the case, the shedding 
A
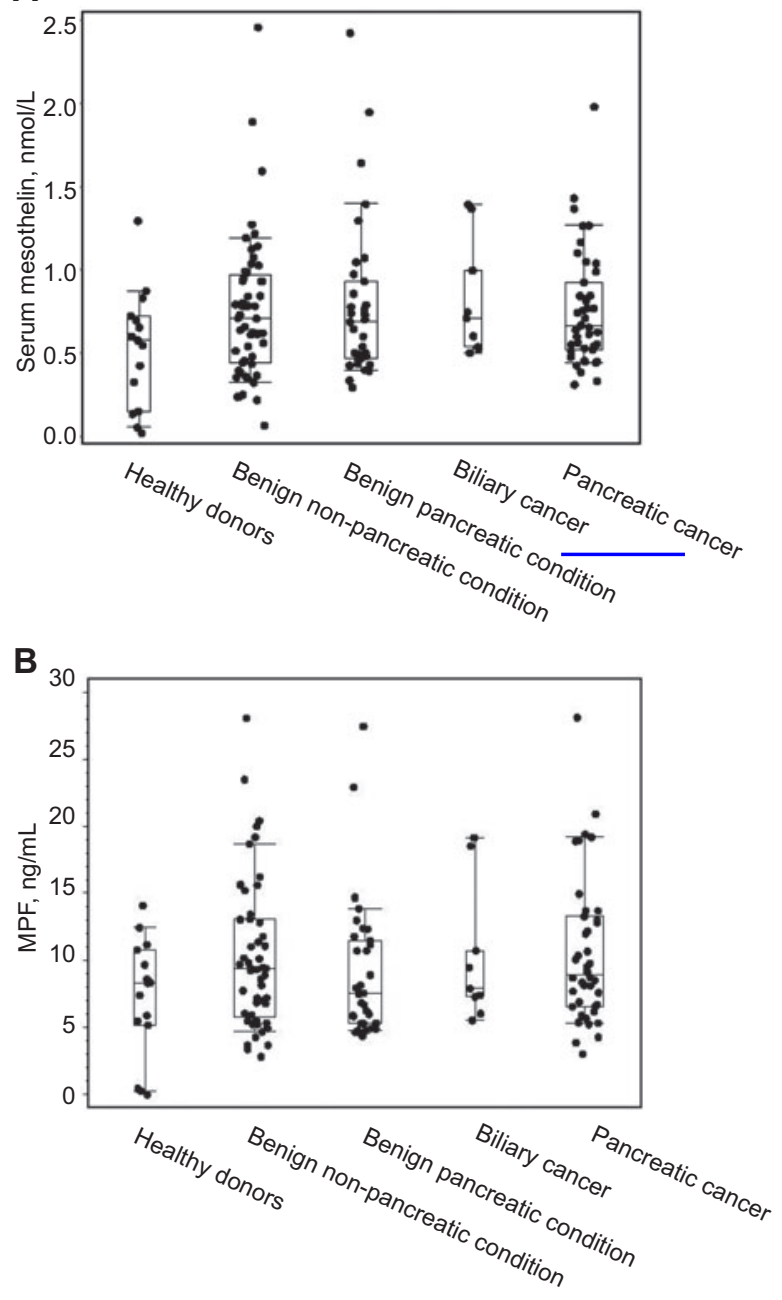

Figure 1 Biomarker concentrations of the study participants.

(A) Serum mesothelin and (B) megakaryocyte potentiating factor (MPF) concentrations of 15 healthy donors, 52 patients with a benign non-pancreatic condition, 33 patients with a benign pancreatic condition, nine with a biliary cancer, and 42 with a pancreatic cancer. For MPF, one outlier $(=59.09 \mathrm{ng} / \mathrm{mL})$, a patient with pancreatic cancer, was not displayed in the graph. Both mesothelin and MPF concentrations did not differ significantly among the different participant groups. Box plot widths vary with the number of participants in each group.

residues could have a different conformation, depending on the malignancy. The development of antibodies that detect different epitopes of mesothelin and MPF might consequently lead to novel insights. Of interest, a previous small study in mesothelioma showed that only approximately half of the patients whose tumors were mesothelin positive had positive mesothelin concentrations in the serum (24). In epithelioid mesothelioma and ovarian carcinoma, a significant number of patients indeed lack elevated mesothelin concentrations in serum or pleural fluid $(8,10,24,25)$. Elucidating the mechanisms behind these observations might also help to shed light on the absence of elevated serum mesothelin concentrations in pancreaticobiliary cancers.
In conclusion, serum mesothelin and MPF concentrations, measured with standard available ELISAs, were not specific for benign or pancreatic disease. In contrast to other mesothelin-overexpressing malignancies, both biomarkers were not elevated in patients with pancreatic or biliary cancers. Neither mesothelin nor MPF consequently appear to be useful biomarkers for these malignancies.

\section{Acknowledgments}

This work was supported by the Intramural Research Program of the NIH, National Cancer Institute, Center for Cancer Research. We thank all study participants and the investigators involved in this study. David J. Liewehr provided technical assistance with the statistical analysis and presentation of data. Yoshiro Kishi and colleagues (Medical \& Biological Laboratories Co., Ltd., Japan) provided the Human MPF ELISA kits.

\section{Conflict of interest statement}

Authors' conflict of interest disclosure: The authors stated that there are no conflicts of interest regarding the publication of this article. Research support played no role in the study design; in the collection, analysis, and interpretation of data; in the writing of the report; or in the decision to submit the report for publication.

Research funding: None declared.

Employment or leadership: None declared.

Honorarium: None declared.

\section{References}

1. Jemal A, Siegel R, Xu J, Ward E. Cancer statistics, 2010. CA Cancer J Clin 2010;60:277-300.

2. Payne SJ, Stebbing J, Wilson P, Slater S. Outcomes in unresectable and locally advanced resected cholangiocarcinoma. Expert Rev Anticancer Ther 2011;11:705-9.

3. Vincent A, Herman J, Schulick R, Hruban RH, Goggins M. Pancreatic cancer. Lancet 2011;378:607-20.

4. Valle J, Wasan H, Palmer DH, Cunningham D, Anthoney A, Maraveyas A, et al. Cisplatin plus gemcitabine versus gemcitabine for biliary tract cancer. N Engl J Med 2010;362:1273-81.

5. Szajda SD, Waszkiewicz N, Chojnowska S, Zwierz K. Carbohydrate markers of pancreatic cancer. Biochem Soc Trans 2011;39:340-3.

6. Hidalgo M. Pancreatic cancer. N Engl J Med 2010;362:1605-17.

7. Locker GY, Hamilton S, Harris J, Jessup JM, Kemeny N, Macdonald JS, et al. ASCO 2006 update of recommendations for the use of tumor markers in gastrointestinal cancer. J Clin Oncol 2006;24:5313-27.

8. Hassan R, Remaley AT, Sampson ML, Zhang J, Cox DD, Pingpank J, et al. Detection and quantitation of serum mesothelin, a tumor marker for patients with mesothelioma and ovarian cancer. Clin Cancer Res 2006;12:447-53.

9. Robinson BW, Creaney J, Lake R, Nowak A, Musk AW, de Klerk $\mathrm{N}$, et al. Mesothelin-family proteins and diagnosis of mesothelioma. Lancet 2003;362:1612-6.

10. Hollevoet K, Nackaerts K, Thimpont J, Germonpré P, Bosquée L, De Vuyst P, et al. Diagnostic performance of soluble mesothelin and megakaryocyte potentiating factor in mesothelioma. Am J Respir Crit Care Med 2010;181:620-5. 
11. Onda M, Nagata S, Ho M, Bera TK, Hassan R, Alexander RH, et al. Megakaryocyte potentiation factor cleaved from mesothelin precursor is a useful tumor marker in the serum of patients with mesothelioma. Clin Cancer Res 2006;12:4225-31.

12. Hassan R, Bera T, Pastan I. Mesothelin: a new target for immunotherapy. Clin Cancer Res 2004;10:3937-42.

13. Hellstrom I, Raycraft J, Kanan S, Sardesai NY, Verch T, Yang Y, et al. Mesothelin variant 1 is released from tumor cells as a diagnostic marker. Cancer Epidemiol Biomarkers Prev 2006;15:1014-20.

14. Argani P, Iacobuzio-Donahue C, Ryu B, Rosty C, Goggins M, Wilentz RE, et al. Mesothelin is overexpressed in the vast majority of ductal adenocarcinomas of the pancreas: identification of a new pancreatic cancer marker by serial analysis of gene expression (SAGE). Clin Cancer Res 2001;7:3862-8.

15. Hassan R, Laszik ZG, Lerner M, Raffeld M, Postier R, Brackett. Mesothelin is overexpressed in pancreaticobiliary adenocarcinomas but not in normal pancreas and chronic pancreatitis. Am J Clin Pathol 2005;124:838-45.

16. Johnston FM, Tan MC, Tan BR Jr., Porembka MR, Brunt EM, Linehan DC, et al. Circulating mesothelin protein and cellular antimesothelin immunity in patients with pancreatic cancer. Clin Cancer Res 2009;15:6511-8.

17. Greene FL, Page DL, Fleming ID, Fritz A, Balch CM, Haller DG, editors. American Joint Committee on Cancer (AJCC) cancer staging manual. 6th ed. New York, NY: Springer, 2002.

18. Beyer HL, Geschwindt RD, Glover CL, Tran L, Hellstrom I, Hellstrom KE, et al. MESOMARK: a potential test for malignant pleural mesothelioma. Clin Chem 2007;53:666-72.
19. Iwahori K, Osaki T, Serada S, Fujimoto M, Suzuki H, Kishi Y, et al. Megakaryocyte potentiating factor as a tumor marker of malignant pleural mesothelioma: evaluation in comparison with mesothelin. Lung Cancer 2008;62:45-54.

20. Hollevoet K, Nackaerts K, Thas O, Thimpont J, Germonpré P, De Vuyst $P$, et al. The effect of clinical covariates on the diagnostic and prognostic value of soluble mesothelin and megakaryocyte potentiating factor. Chest Epub 2011 Jul 7. PMID: 21737491.

21. Einama T, Kamachi H, Nishihara H, Homma S, Kanno H, Takahashi K, et al. Co-expression of mesothelin and CA125 correlates with unfavorable patient outcome in pancreatic ductal adenocarcinoma. Pancreas 2011;40:1276-82.

22. Creaney J, Francis RJ, Dick IM, Musk AW, Robinson BW, Byrne MJ, et al. Serum soluble mesothelin concentrations in malignant pleural mesothelioma: relationship to tumor volume, clinical stage and changes in tumor burden. Clin Cancer Res 2011;17:1181-9.

23. Hollevoet K, Nackaerts K, Gosselin R, De Wever W, Bosquée L, De Vuyst P, et al. Soluble mesothelin, megakaryocyte potentiating factor, and osteopontin as markers of patient response and outcome in mesothelioma. J Thorac Oncol 2011;6:1930-7.

24. Creaney J, van Bruggen I, Hof M, Segal A, Musk AW, de Klerk $\mathrm{N}$, et al. Combined CA125 and mesothelin levels for the diagnosis of malignant mesothelioma. Chest 2007;132:1239-46.

25. Yamada S, Tabata C, Tabata R, Fukuoka K, Nakano T. Clinical significance of pleural effusion mesothelin in malignant pleural mesothelioma. Clin Chem Lab Med 2011;49:1721-6. 